\title{
Study of Epoxy Bonding
}

\author{
Xiaohua Zhou, Zhiyuan Liu, Hui Xing \\ Beijing Institute of Space Mechanics \& Electricity, Beijing, China \\ Email: zxh_2102@126.com
}

How to cite this paper: Zhou, X.H., Liu, Z.Y. and Xing, H. (2021) Study of Epoxy Bonding. Journal of Computer and Communications, 9, 214-221. https://doi.org/10.4236/jcc.2021.96013

Received: June 1, 2021

Accepted: June 27, 2021

Published: June 30, 2021

\begin{abstract}
Bonding is used widely when the optical glasses are connected with metal structures. It plays a very important role in the design of space telescope. Improper bonding may induce great surface figure error for optics in telescope. Milbond and EC2216 are the most popular epoxies that are used in optics. Bubbles usually exist in the epoxy when two components are mixed or translated to syringe. In this paper, the methods and experiments of adhesive mixture and injection in vacuum environment were explained to reduce the bonding surface figure of the mirror. The results show that adhesive mixture and injection in vacuum environment can dramatically decrease the bubble in the epoxy and greatly reduce the bonding surface figure error.
\end{abstract}

\section{Keywords}

Epoxy, Bonding, Vacuum, Milbond, EC 2216

\section{Introduction}

Bonding is used widely when the optical glasses are connected with metal structures. It plays a very important role in the design of space telescope.

Compared with RTV adhesive, epoxy adhesive has the advantages of high bonding strength and small linear expansion coefficient. Although bonding with RTV adhesive can minimize the change of the mirror surface figure error, the bonding strength of RTV adhesive is only about $1 \sim 2 \mathrm{MPa}$ [1] [2] [3]. And the thickness of the adhesive layer is thicker, generally greater than $0.5 \mathrm{~mm}$, and the linear expansion coefficient is more than $2 \times 10^{-4} \mathrm{~K}^{-1}$, and the surface figure error is sensitive to changes in the temperature environment. Due to the special mechanical and space environment experienced by the space mirror, the bonding strength between the mirror and the metal structure is required to be sufficient, in many cases, epoxy is the best choice to bond the mirror and the mounting structure. 
There are many factors that affect the surface figure error of the mirror surface in the bonding process, such as the mismatching of the linear expansion coefficient of the bonding material, the solidification and shrinkage of the adhesive layer, and the control of the bonding process. The space mirror needs to go through the vacuum thermal alternating environment, so it is very important to control the bonding process to reduce the bubbles in the adhesive layer. Bubbles in the adhesive layer degrade the surface figure in vacuum.

Milbond and EC2216 are the most popular epoxies that are used in optics. In this paper, the methods and experiments of adhesive mixture and injection were explained to reduce the bonding surface figure of the mirror

\section{Problems of Epoxy Mixing}

In order to control the thickness and the shape of adhesive layer, epoxy adhesive is usually injected by syringe. Milbond and EC2216 are both composed of two components that need to be thoroughly mixed and loaded into a syringe before use. The viscosity of these two kinds of glue is high, and the fluidity are not very good. It is easy to mix with air inside during the process of mixing in the atmosphere. In the process of curing, the bubbles cannot come out from the adhesive in time. The Figure 1 is the specimens of Milbond. The required size of the test piece is obtained by removing the surface adhesive layer. It can be clearly seen that there are many bubbles in the specimen.

Bubbles affect not only the bonding strength, but also the mechanical properties of the adhesive layer. The adhesive used in the space telescope needs to be tested in a vacuum. When the gas in the adhesive layer diffuses in the vacuum environment, it will affect the stress distribution in the adhesive layer and easily lead to the change of the surface figure. In order to keep the accuracy of the mirror surface, the glue used in the space telescope should remove the bubbles as much as possible.

\section{Experiments of Mixing Glue in Vacuum}

In order to reduce the air entry in the process of glue mixing and glue transformation to syringe, a set of vacuum and glue device is manufactured, as shown in the Figure 2. A vacuum tank is about $\varnothing 350 \mathrm{~mm}$, and height is about $500 \mathrm{~mm}$

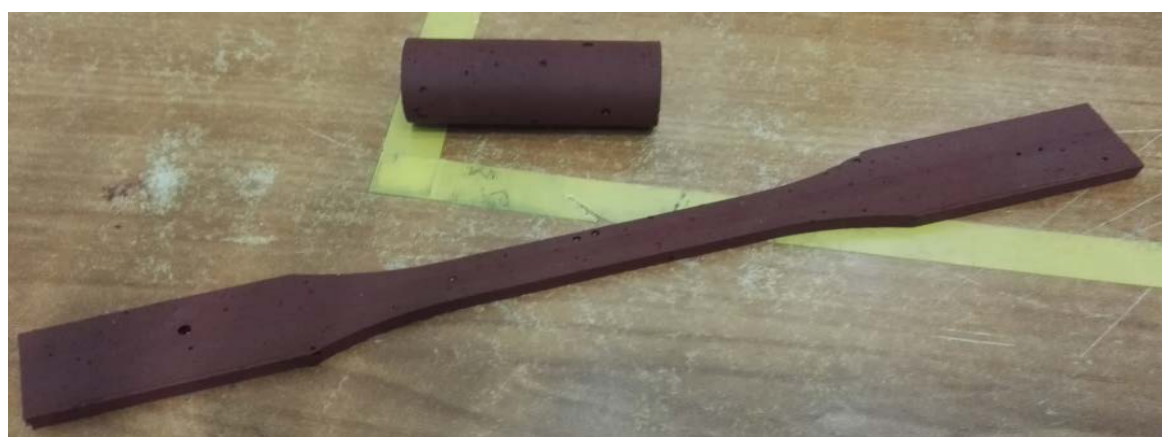

Figure 1. Bubbles in specimen. 
with cover plate, external pressure gauge and vacuum pump are connected to the vacuum tank. The pressure gauge is used to detect the pressure inside the tank. Cover plate is transparent plate, it is convenient to observe the change of glue from the outside. Two components of the epoxy adhesive are poured into the container in proportion in the tank. A mixing device which is composed of a DC motor and an agitator is fixed on the top of the container. The DC motor is drived by the $24 \mathrm{~V}$ DC power through the canning plug. The diameter of the syringe is usually about $20-30 \mathrm{~mm}$. It's too small to install the mixer. the mixing glue and transformation of the glue to syringe are carried out in two separate steps. After the glue is mixed in vacuum, the mixing container is placed on the cover plate outside the vacuum tank. There is a small hole at the bottom of the mixing container, and the mixed glue flows through the cover plate into the syringe at the bottom through the hole, and the syringe is fixed in the vacuum tank through the bracket, as shown in Figure 3. Under the action of external pressure and assumed pressure, the glue flows into the vacuum tank and enters the syringe, realizing the glue is loaded into the syringe in the vacuum environment.

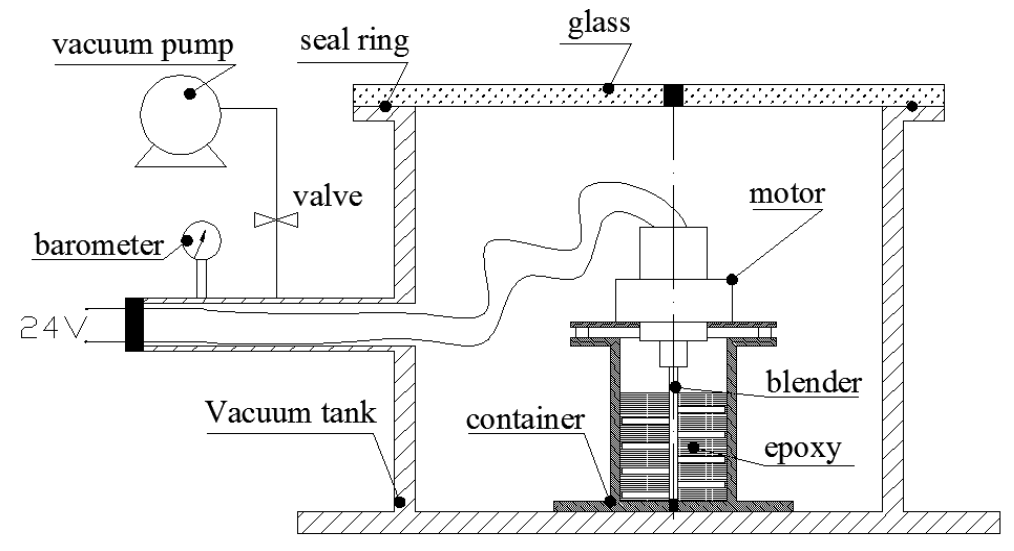

Figure 2. Mixture epoxy in vacuum.

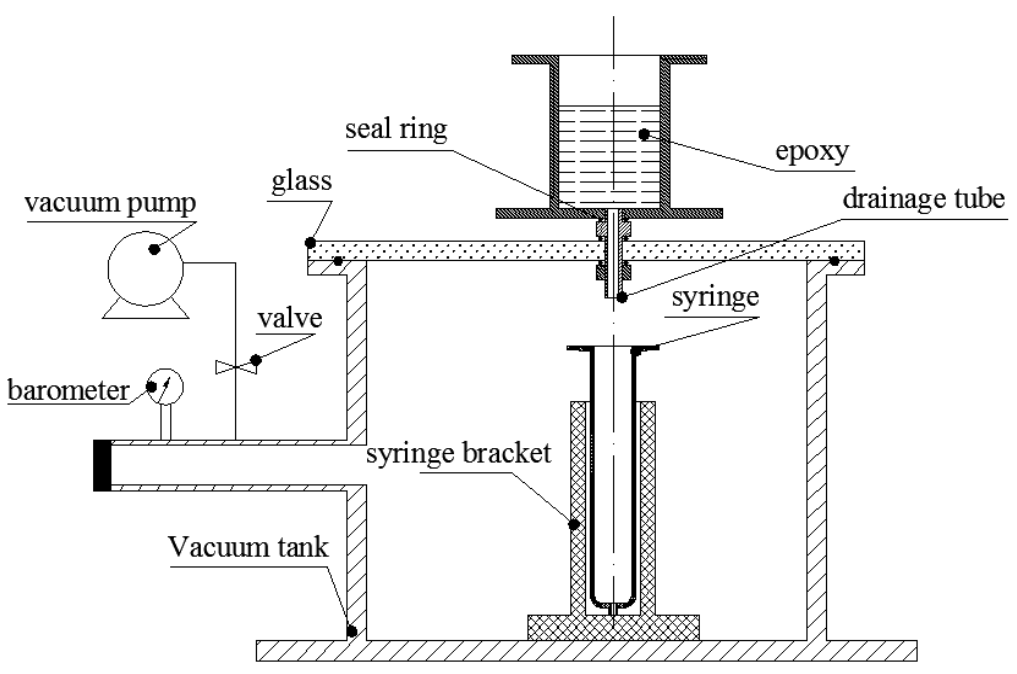

Figure 3. Transformation epoxy to syringe in vacuum. 
In the process of vacuum mixing, the state of the epoxy change can be observed through the glass cover plate. When the pressure is down to $10 \mathrm{~Pa}$, the volume of the epoxy begins to expand, there are lots of bubbles emerging from the epoxy. When the pressure recovers, the volume of the adhesive contracts and the bubbles in the adhesive quickly burst. The pressure is down to $0.1 \mathrm{~Pa}$ and kept for 5 minutes, then return to normal again, 3 times later, After scraping off the incomplete solidified adhesive layer with a shovel knife, the bubble of the adhesive decreases obviously. This is an effective way to decrease the gas that induced by epoxy mixture and transformation to syringe.

There is one thing that exceeding expected, that is after a number of vacuumrecover-pressure cycles, Bubbles still continue to escape out of the glue. Further experiments show that there will be lots of bubbles flee from the separated components even if they are not mixed in the vacuum. That means that there are volatile molecules in each components and they will be escape from the resin in low air pressure.

We decide to find out what will be happen after mixture if each component stay in vacuum for enough time. Two experiments carried out to verify the properties of the epoxy after fully degassing in component state and mixture and transformation in vacuum. One comparative experiment of the bonding strength was taken; another experiment is the comparative surface figure error changes test.

\section{Comparative Bonding Strength Experiment}

Comparative bonding strength test is done under the same bonding and curing conditions, the thickness of the adhesive layer is controlled to $0.05 \mathrm{~mm}$. The glass used in the strength test is ULE, and the metal is titanium. 10 tensile strength test pieces of Milbond and EC2216 were made.

The test results after stretching are shown in Table 1 and Table 2. In the curing

Table 1. Tensile bonding strength of Milbond mixed in vacuum.

\begin{tabular}{cc}
\hline No. & Tensile bonding strength $(\mathrm{MPa})$ \\
\hline 1 & 3.14 \\
2 & 4.58 \\
3 & 3.78 \\
4 & 4.07 \\
5 & 4.21 \\
6 & 6.96 \\
7 & 5.71 \\
8 & 3.72 \\
9 & 5.12 \\
10 & 3.58 \\
average & 4.49 \\
\hline
\end{tabular}


Table 2. Tensile bonding strength of EC2216 mixed in vacuum.

\begin{tabular}{cc}
\hline No. & Tensile bonding strength (MPa) \\
\hline 1 & 4.65 \\
2 & 4.43 \\
3 & 3.97 \\
4 & 4.31 \\
5 & 3.81 \\
6 & 3.78 \\
7 & 5.15 \\
8 & 4.73 \\
9 & 4.35 \\
10 & 5.17 \\
average & 4.43 \\
\hline
\end{tabular}

Table 3. Bonding strength in the tensile test.

\begin{tabular}{ccc}
\hline & $\begin{array}{c}\text { Tensile bonding } \\
\text { strength }(\mathrm{MPa})\end{array}$ & $\begin{array}{c}\text { Tensile bonding strength Degassing } \\
\text { in vacuum }(\mathrm{MPa})\end{array}$ \\
\hline Milbond & 8.96 & 4.49 \\
EC2216 & 12 & 4.43 \\
\hline
\end{tabular}

process, the curing time of the adhesive layer after complete degassing is much longer than without degassing. At room temperature and atmosphere pressure in the laboratory, it is confirmed that all the adhesives are cured completely one month after bonding. The degassing adhesive layer is much softer than the one without degassing after glassify. Based on the former tensile bonding strength test in normal condition, the average bonding tensile strength of Milbond decreased from 8.96 MPa without degassing to 4.49 $\mathrm{MPa}$ with degassing for milbond, and the bonding strength decreased by $49.9 \%$. The bonding strength of EC2216 decreases from 12 MPa to 4.43 MPa, as shown in Table 3, and the bonding strength decreases by $63.1 \%$. Analysis of component show that some components evaporate during mixture. The inter-connection reactions are not enough that lead to the tensile bonding strength decrease.

Although the bonding strength is very important factor, it can be compensated by bonding area. The surface figure change after bonding is the key factor for epoxy bonding in space telescope.

\section{Comparative Surface Figure Error Change Experiment}

Comparative surface figure error change experiment is to test the surface figure error change at the same condition by epoxy with degassing and without degassing. Employed test specimen are made from $\varnothing 70 \mathrm{~mm}$ thickness of $30 \mathrm{~mm}$ ULE glass with an face polished, surface figure is processed to better than $0.014 \lambda$. A $\varnothing 20 \mathrm{~mm}$ invar pipe which is about $60 \mathrm{~mm}$ in length is bonded to the hole which 
axis is perpetual to axis of the ULE glass. The invar and ULE and epoxy between the same kind of specimens have the same properties. The specimens are shown in Figure 4 and Figure 5. Due to the limit of the testing budget, the number of test specimens in this test is only two pieces of each degassing epoxies, while the number of test specimens are three for without degasing ones. The full curing period is one month. After a month we started testing the surface figure error of the mirror. In order to examine the influence of temperature cycle and vacuum environment on the mirror shape, the vacuum environment and temperature cycle were tested separately for degasing epoxies specimens.

Table 4 and Table 5 show surface figure error change using degassing epoxies. Table 6 and Table 7 show the surface figure error change using without degassing epoxies [4]. As can be seen from Table 4 to Table 7, comparing degassing and without degasing, the average surface figure error increment change

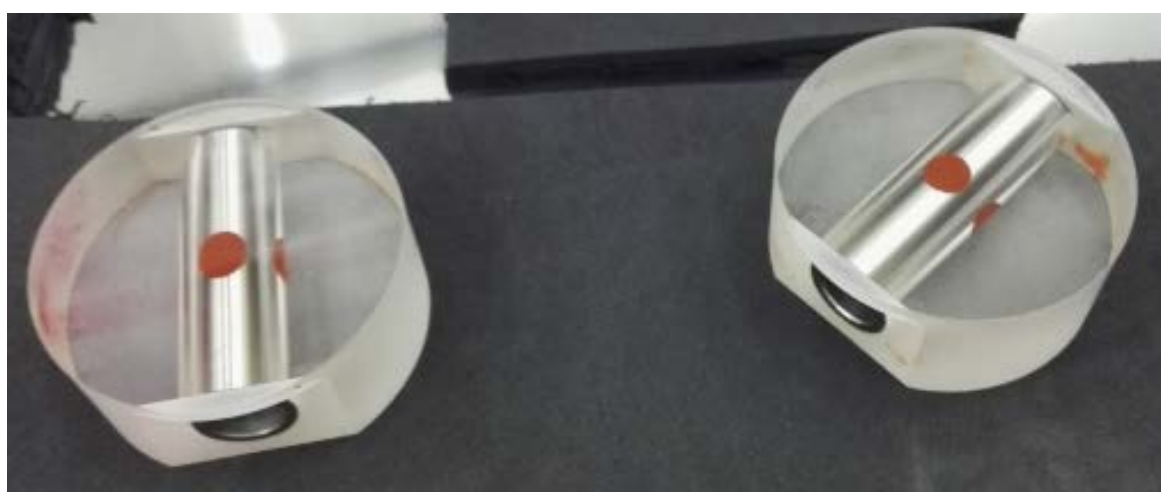

Figure 4. Surface figure specimen of Milbond.

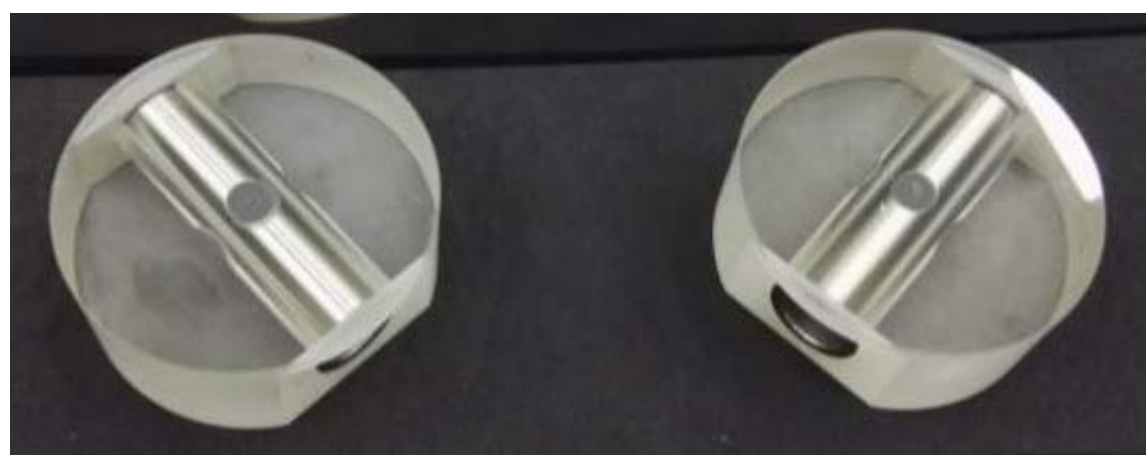

Figure 5. Surface figure specimen of EC2216.

Table 4. Surface figure error change using degasing Milbond.

\begin{tabular}{ccc}
\hline & \multicolumn{2}{c}{ Milbond } \\
\cline { 2 - 3 } & $1 \#$ & 2\# \\
\hline Surface figure error before bonding $(\lambda)$ & 0.013 & 0.015 \\
Surface figure error one month after bonding $(\lambda)$ & 0.035 & 0.036 \\
Surface figure error after 24 h vacuum deflating at room temperature $(\lambda)$ & 0.034 & 0.034 \\
Surface figure error after three thermal cycles test $(\lambda)$ & 0.085 & 0.097 \\
\hline
\end{tabular}


Table 5. Surface figure error change using degasing EC2216.

\begin{tabular}{ccc}
\hline & \multicolumn{2}{c}{ EC2216 } \\
\cline { 2 - 3 } & $3 \#$ & 4\# \\
\hline Surface figure error before bonding $(\lambda)$ & 0.009 & 0.012 \\
Surface figure error one month after bonding $(\lambda)$ & 0.016 & 0.025 \\
Surface figure error after 24 h vacuum deflating at room temperature $(\lambda)$ & 0.022 & 0.025 \\
Surface figure error after three thermal cycles test $(\lambda)$ & 0.038 & 0.040 \\
\hline
\end{tabular}

Table 6. Surface figure error change using without degassing Milbond.

\begin{tabular}{cccc}
\hline & \multicolumn{3}{c}{ Milbond } \\
\cline { 2 - 4 } & $1 \#$ & $2 \#$ & $3 \#$ \\
\hline Surface figure error before bonding $(\lambda)$ & 0.011 & 0.015 & 0.011 \\
Surface figure error of 9 days after bonding $(\lambda)$ & 0.040 & 0.039 & 0.040 \\
Surface figure error after vacuum and three thermal cycles $(\lambda)$ & 0.255 & 0.219 & 0.211 \\
\hline
\end{tabular}

Table 7. Surface figure error change using without degassing EC2216.

\begin{tabular}{cccc}
\hline & \multicolumn{3}{c}{ EC2216 } \\
\cline { 2 - 4 } & $4 \#$ & $5 \#$ & $6 \#$ \\
\hline Surface figure error before bonding $(\lambda)$ & 0.014 & 0.016 & 0.016 \\
Surface figure error of 9 days after bonding $(\lambda)$ & 0.103 & 0.074 & 0.115 \\
Surface figure error after vacuum and three thermal cycles $(\lambda)$ & 0.127 & 0.089 & 0.131 \\
\hline
\end{tabular}

from $0.1 \lambda$ to $0.028 \lambda$ for EC2216 adhesive after vacuum and thermal cycle test, reduced by $72 \%$. After vacuum degasses curing and vacuum and thermal cycle tests, comparing degassing and without degasing, the average surface figure error increment changes from $0.216 \lambda$ to $0.077 \lambda$ for Milbond, which decreases about $64.4 \%$. It can also be seen that the thermal environment has a much greater influence on the surface figure error than the vacuum environment.

\section{Conclusion}

In this paper, the methods of epoxies mixture and injection in vacuum environment were explained to reduce surface figure error of the mirror. The comparative bonding strength tests and surface figure error test of two epoxies were carried out. Although the bonding strength decreased after degassing in vacuum, the bonding influence on the surface figure error was greatly reduced.

\section{Acknowledgements}

The work is supported by Foundation of State Administration of Science, Technology and Industry for National Defense (D040106), and also supported by Beijing Institute of Space \& Electricity and Beijing Key Laboratory of Advanced Optical Remote Sensing Technology. 


\section{Conflicts of Interest}

The authors declare no conflicts of interest regarding the publication of this paper.

\section{References}

[1] Park, Y.J., Lim, D.H., Kim, H.J., et al. (2009) UV- and Thermal-Curing Behaviors of Dual-Curable Adhesives Based on Epoxy Acrylate Oligomers. International Journal of Adhesion and Adhesives, 29, 710-717. https://doi.org/10.1016/j.ijadhadh.2009.02.001

[2] Simic, S., Dunjic, B., Tasic, S., et al. (2008) Synthesis and Characterization of Interpenetrating Polymer Networks with Hyperbranched Polymers through Thermal-UV Dual Curing. Progress in Organic Coatings, 263, 43-48.

https://doi.org/10.1016/j.porgcoat.2008.04.006

[3] Yuan, H., Lu, X. and Zeng, Z. (2005) Allyl Ether Modified Unsaturated Polyesters for UV/Air Dual-Curable Coatings. I: Synthesisand Characterization of the Oligomers and Their Cured Films. Journal of Applied Polymer Science, 92, 2765-2770. https://doi.org/10.1002/app.20273

[4] Zhou, X.H. (2019) Epoxy Selection for Reflect Mirror Assembly in Space Remote Sensor. Spacecraft Recovery \& Remote Sensing, 40, 65-70. 